\title{
Thermoelectric Properties of Silver Antimonate with Mixed Valency of Antimony
}

\author{
Kensuke Ozawa1, Hirofumi Kakemoto ${ }^{2}$, Hiroshi Irie ${ }^{1,2^{*}}$ \\ ${ }^{1}$ Interdisciplinary Graduate School of Medicine and Engineering, University of Yamanashi, Yamanashi, Japan \\ ${ }^{2}$ Clean Energy Research Center, University of Yamanashi, Yamanashi, Japan \\ Email: ${ }^{\star h i r i e @ y a m a n a s h i . a c . j p ~}$
}

How to cite this paper: Ozawa, K., Kakemoto, H. and Irie, H. (2017) Thermoelectric Properties of Silver Antimonate with Mixed Valency of Antimony. Journal of Materials Science and Chemical Engineering, 5, 121-128.

http://dx.doi.org/10.4236/msce.2017.51016

Received: December 14, 2016

Accepted: January 13, 2017

Published: January 16, 2017

Copyright $\odot 2017$ by authors and Scientific Research Publishing Inc. This work is licensed under the Creative Commons Attribution International License (CC BY 4.0).

http://creativecommons.org/licenses/by/4.0/ (c) (i) Open Access

\begin{abstract}
Silver $(\mathrm{Ag})$ and silver antimonate $\left(\mathrm{AgSbO}_{3}\right)$ composites with different amounts of $\mathrm{Sb}^{3+}$ were synthesized by normal sintering with the aim of realizing a thermoelectric material. The electrical conductivity $(\sigma)$ increased in the sample containing larger amount of $\mathrm{Sb}^{3+}$, whereas Seebeck coefficient $(S)$ decreased. Producing $\mathrm{Sb}^{3+}$ caused the generation of oxygen vacancies in the material, and thus the corresponding donor levels are created in the bandgap, providing more conduction electrons. The conductive Ag particles would contribute to the conduction path as bypasses for carrier transport. The thermal conductivity $(\kappa)$ was slightly lower in the presence of $\mathrm{Ag}$ defects in $\mathrm{AgSbO}_{3}$.
\end{abstract}

\section{Keywords}

Thermoelectric Material, Silver Antimonate, Seebeck Coefficient, Electrical Conductivity, Thermal Conductivity

\section{Introduction}

Thermoelectric (TE) materials have been attracting attention due to their potential for recycling energy using exhausted heat through the thermal-electric conversion effect, generating clean energy without polluting the environment. Recently, several oxides have been recognized as potential thermoelectric materials [1] [2] [3] [4]. TE conversion efficiency is represented by a dimensionless figure of merit, $Z T=S \sigma T / \kappa$, where $S, \sigma, \kappa$, and $T$ are the Seebeck coefficient, electrical conductivity, thermal conductivity, and absolute temperature, respectively. From these equations, large $S$ and $\sigma$ values and a low $\kappa$ are necessary for high TE performance.

It is known that typical oxides have a low mobility $(\mu)$ and high $\kappa$ (particularly, $\kappa_{\mathrm{ph}}$, which is the thermal conductivity mediated by phonons), originating from 
their ionic bonding between light atoms and high electronegativity of oxygen. Different from these oxides, silver antimonite $\left(\mathrm{AgSbO}_{3}\right)$, which has a defect pyrochlore structure composed of linear chains of $\mathrm{AgO}_{6}$ and $\mathrm{SbO}_{6}$, possesses rather high $\mu$ originating from its highly-dispersed valence band and conduction band composed of $\mathrm{Ag} 5 \mathrm{~s}$ and $\mathrm{Sb} 5 \mathrm{~s}$ orbitals, respectively [5] [6]. In addition, $\mathrm{AgSbO}_{3}$ has low stacking density and thus its $\kappa_{\mathrm{ph}}$ is significantly lower than those of other oxides [7] [8]. So, $\mathrm{AgSbO}_{3}$ has been investigated as a candidate n-type TE material, e.g., by addition of $\mathrm{CuO}$ [5] and by using a spark plasma sintering (SPS) method to prepare dense $\mathrm{AgSbO}_{3}$ [7]. According to Wiggers et al., aggregated Ag islands are formed in the thermally treated $\mathrm{AgSbO}_{3}$, causing the increase in $\sigma$ by the electron hopping between such metallic islands [9]. However, the previous studies, regarding TE properties of $\mathrm{AgSbO}_{3}$, did not mention the existence of metallic Ag. Moreover, the valency of $\mathrm{Sb}\left(\mathrm{Sb}^{3+}\left(4 \mathrm{~d}^{10}\right), \mathrm{Sb}^{5+}\left(4 \mathrm{~d}^{10} 5 \mathrm{~s}^{2}\right)\right)$ should affect $\sigma$ due to generation of oxygen vacancy in the presence of $\mathrm{Sb}^{3+}$; however such discussion has not been performed. Thus, in the present paper, we characterize the $\mathrm{AgSbO}_{3}$ in details, prepared by the combination of a solid state reaction (SSR) method and a nitric acid $\left(\mathrm{HNO}_{3}\right)$ treatment [10]. In addition, the existence of metallic silver and the ratio of $\mathrm{Sb}^{3+} / \mathrm{Sb}^{5+}$ in $\mathrm{AgSbO}_{3}$ are discussed in connection with $\sigma, S$, and $\kappa$.

\section{Experimental}

$\mathrm{AgSbO}_{3}$ was synthesized by SSR using $\mathrm{Ag}_{2} \mathrm{O}$ (>99.0\%, Kanto Kagaku) and $\mathrm{Sb}_{2} \mathrm{O}_{3}$ $(>98.0 \%$, Kanto Kagaku) as raw materials. The raw materials were weighed to the stoichiometric molar ratio, and were mixed by ball milling $(200 \mathrm{rpm}$, milling rate) for $20 \mathrm{~h}$, and then the mixed powder was calcined at $900^{\circ} \mathrm{C}$ for $2 \mathrm{~h}$. The partial calcined powder was uniaxially pressed into a rectangular pellet with the dimensions $\sim 4 \times 5 \times 20 \mathrm{~mm}$, followed by calcination in an electric furnace (HPM-1N, AS-ONE) in air at $900^{\circ} \mathrm{C}$ for $2 \mathrm{~h}$ (denoted by NS). The remaining calcined powder was washed by $\mathrm{HNO}_{3}(5.0 \mathrm{~mol} / \mathrm{L}$, Kanto Kagaku), then dried at $90^{\circ} \mathrm{C}$ for $20 \mathrm{~h}$. The obtained powder was pressed into a pellet followed by calcination in the electric furnace under the same conditions, and obtained sample was denoted by NAT-NS.

The obtained calcined powders and sintered bodies, after grounding powders and pulverizing bodies, respectively, into fine powders were characterized by conventional X-ray diffraction analysis (XRD) with $\mathrm{CuK}_{\alpha}$ radiation (PANalytical, $\mathrm{PW}-1700)$. Synchrotron radiation powder diffraction patterns were obtained at BL02B2 in SPring-8 after pulverizing bodies into fine powders. The patterns were collected in the $2 \theta$ range of $3^{\circ}-73^{\circ}$ in a step scanning mode, with a step length of $0.01^{\circ}$. The Rietveld analyses using a RIETAN-FP software (a multipurpose pattern-fitting system) were performed on the synchrotron radiation diffraction patterns. For the analysis, the space groups Fd3m (cubic, No. A-227) and Fm-3m (cubic, No. A-225) were used as the starting structural model of $\mathrm{AgSbO}_{3}(a=10.23 \mathrm{~nm})$ and $\mathrm{Ag}(a=4.0857 \mathrm{~nm})$. A scanning electron microscope (SEM; JSM-6500F, JEOL) was used to observe the morphology of cross-sectional 
surfaces of the samples. The valency of Ag and Sb ions was evaluated by X-ray photoelectron spectroscopy (XPS; Axis-Ultra, Shimadzu).

To measure $\sigma$ and $S$, either two or four Pt wires, respectively, were attached by an Ag-conductive region to the prepared rectangular pellet samples. The temperature dependence of $S$ (Yokogawa, Model 7563) with a temperature gradient of $5-20 \mathrm{~K}$ and that of $\sigma$ (Agilent Technologies, E5273A) were measured from $473 \mathrm{~K}$ to $873 \mathrm{~K}$ by conventional two-probe steady-state and four-probe methods, respectively. We then calculated the power factor $(P F)$, as expressed by $S^{2} \sigma$, for each sample. A modified Harman method was used to measure $Z T$ at $600^{\circ} \mathrm{C}$ [11] and then we calculated $\kappa$ by using the equation, $Z T=S^{2} \sigma T / \kappa$.

\section{Results and Discussion}

\subsection{Characterizations}

The observed diffraction patternsfor NS and NAT-NS by synchrotron radiation are shown in Figure 1(a) and Figure 1(b), respectively, and indicated it adopted double phases of cubic $\mathrm{AgSbO}_{3}$ and $\mathrm{Ag}$. Not shown here, but calcined $\mathrm{AgSbO}_{3}$ indicated the homogeneous defect pyrochlore structure by the conventional $\mathrm{XRD}$. The detection of $\mathrm{Ag}$ was reasonably expected as $\mathrm{Ag}_{2} \mathrm{O}$ is thermally decom-
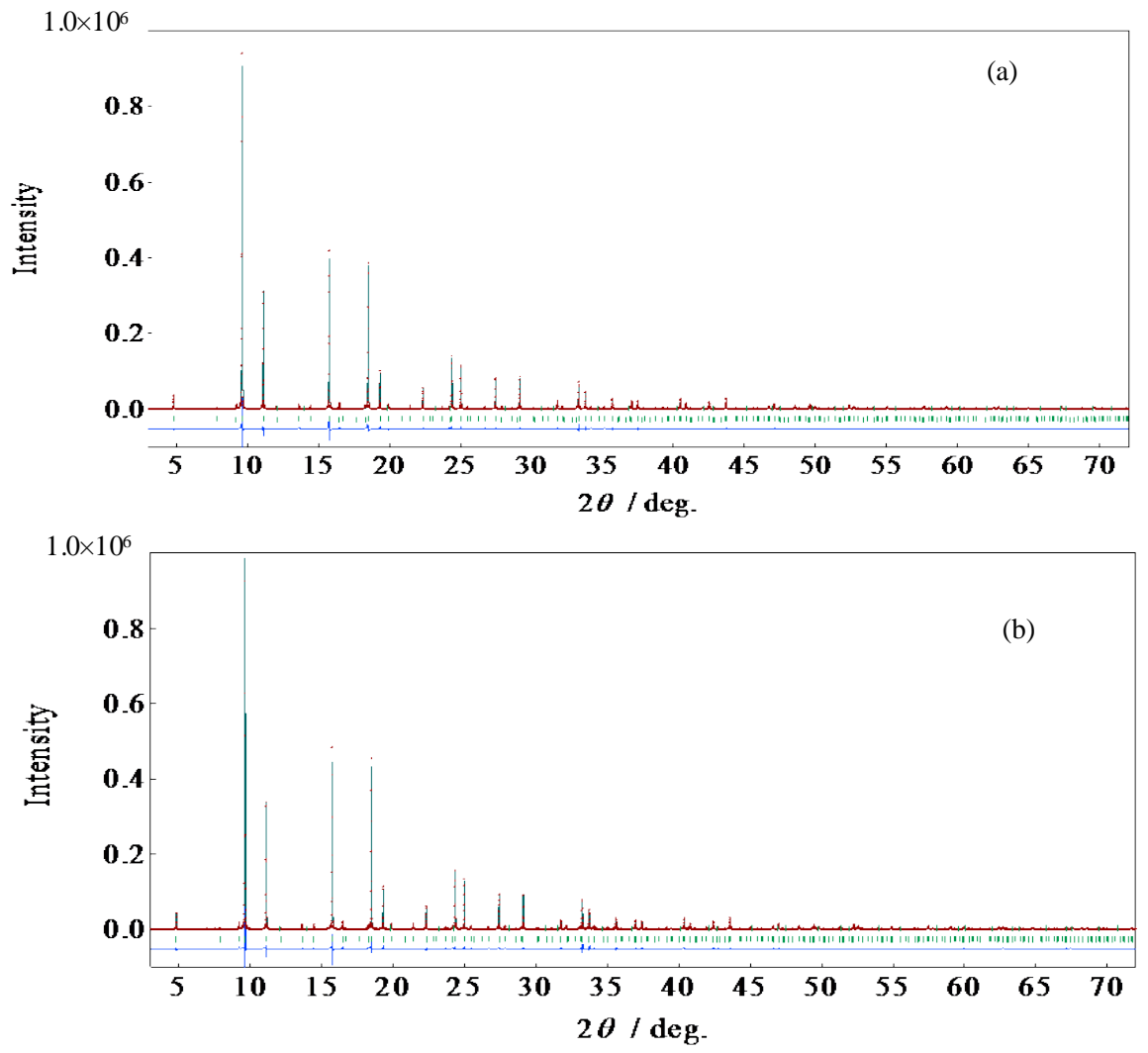

Figure 1. Measured synchrotron radiation diffraction patterns and patterns calculated by Rietveld analysis for the prepared samples, (a) NS and (b) NAT-NS. The dotted red plots, black lines, and blue lines represent measured patterns, calculated patterns, and the differences between the measured and calculated patterns, respectively. Crystallographic data determined by the Rietveld analysis are shown in Table 1. 
posed to $\mathrm{Ag}$ at $\sim 280^{\circ} \mathrm{C}$. Figure 1 also includes the calculatedpattern involving $\mathrm{AgSbO}_{3}$ and $\mathrm{Ag}$, and the difference between the observed and calculated values. The molar percentages of $\mathrm{AgSbO}_{3}$ and $\mathrm{Ag}$ in both samples, their lattice parameters, and their compositions were determined by Rietveld refinement and are summarized in Table 1 . In the NS sample, the $\mathrm{Ag} / \mathrm{Sb}$ ratio is unity, in good agreement with the stoichiometric ratio $\left(\mathrm{Ag}_{1.00} \mathrm{Sb}_{1.00} \mathrm{O}_{3}\right)$, although $\mathrm{Ag}$ exists separately in spite of the stoichiometric ratio of starting materials, $\mathrm{Ag}_{2} \mathrm{O}$ and $\mathrm{Sb}_{2} \mathrm{O}_{3}$. This is reasonable because $\mathrm{Sb}$ element volatilized during sintering [5] [8]. In contrast, in the NAT-NS sample, the $\mathrm{Ag} / \mathrm{Sb}$ ratio is less than unity $\left(\mathrm{Ag}_{0.98} \mathrm{Sb}_{1.00} \mathrm{O}_{3}\right)$, reflecting the $\mathrm{HNO}_{3}$ treatment which extracted $\mathrm{Ag}$ from the $\mathrm{AgSbO}$ lattice, causing the Ag defect in $\mathrm{AgSbO}_{3}$. The amounts of the separated Ag in both samples were observed to be similar. The relative densities of NS and NAT-NS pellets were also quite similar, 58.9 and 56.6\%, respectively (Table 2). Those values were small, $\sim 60 \%$ of the theoretical ones, which is considered to be attributable to the evaporation of $\mathrm{Sb}[5]$.

SEM images of the fracture surfaces of NS and NAT-NS are shown in Figure 2(a) and Figure 2(b), respectively. The NS and NAT-NS powders, with particle sizes of $\sim 100-200 \mathrm{~nm}$ in diameter, appeared to be aggregated, indicating that both of them were porous. The binding energies corresponding to $A g 3 \mathrm{~d}_{5 / 2}$ of $\mathrm{Ag}^{0}(\mathrm{Ag}), \mathrm{Ag}^{+}\left(\mathrm{Ag}_{2} \mathrm{O}\right)$, and $\mathrm{Ag}^{2+}(\mathrm{AgO})$ are quite similar, and are 368.0 - 368.3, 367.6 - 367.8, and $367.3-367.4 \mathrm{eV}$, respectively [12]. Since all the energies are in such a narrow range, it is quite difficult to perform a quantitative analysis of the Ag oxidation states from a deconvolution analysis of XPS spectra with high certainty. However, qualitatively, as shown in Figure 3(a), the spectrum peak of $\mathrm{HNO}_{3}$-treated sample (NAT-NS) shifted to the higher binding energy side (inset in Figure 3(a)), and also the spectrum had tailing in the lower binding energy region. These observations indicate that the $\mathrm{HNO}_{3}$-treated sample has more $\mathrm{Ag}$

Table 1. Crystallographic data determined by the Rietveld analysis.

\begin{tabular}{lcc}
\hline Sample & NS & NAT-NS \\
\hline$R_{w p}$ & 8.90 & 10.1 \\
$S$ & 6.19 & 6.86 \\
Ag content/mol\% & 0.79 & 0.83 \\
Lattice parametrt of $\mathrm{Ag}, \mathrm{a} / \mathrm{nm}$ & 4.078 & 4.081 \\
Lattice parametrt of $\mathrm{AgSbO}, \mathrm{a} / \mathrm{nm}$ & 10.22 & 10.25 \\
Composition of $\mathrm{AgSbO}_{3}{ }^{\mathrm{a}}$ & $\mathrm{Ag}_{1.00} \mathrm{Sb}_{1.00} \mathrm{O}_{3}$ & $\mathrm{Ag}_{0.98} \mathrm{Sb}_{1.00} \mathrm{O}_{3}$ \\
\hline
\end{tabular}

a. Estimated from the occupancies of $\mathrm{Ag}$ and $\mathrm{Sb}$ in $\mathrm{AgSbO}_{3}$.

Table 2. Characterization data of NS and NAT-NS, SPS samples.

\begin{tabular}{ccc}
\hline Sample & NS & NAT-NS \\
\hline Relative density $\%$ & 58.9 & 56.6 \\
$\mathrm{Sb}^{3+} / \mathrm{Sb}^{5+}$ ratio & 1.5 & 0.23 \\
\hline
\end{tabular}



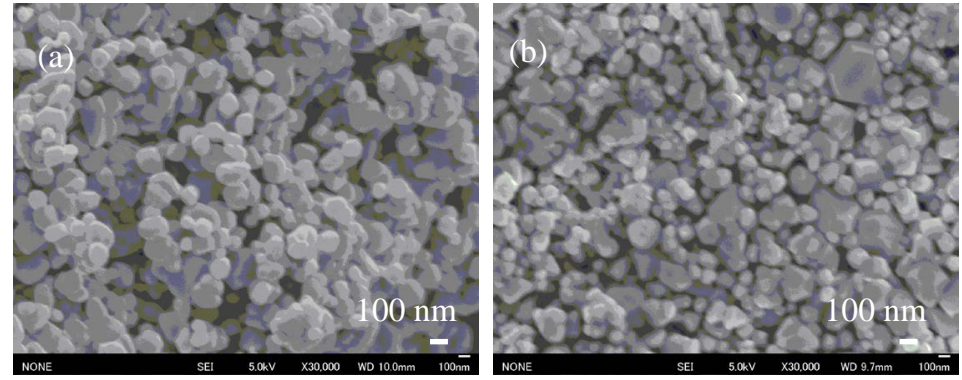

Figure 2. Cross-sectional SEM images of (a) NS and (b) NAT-NS.
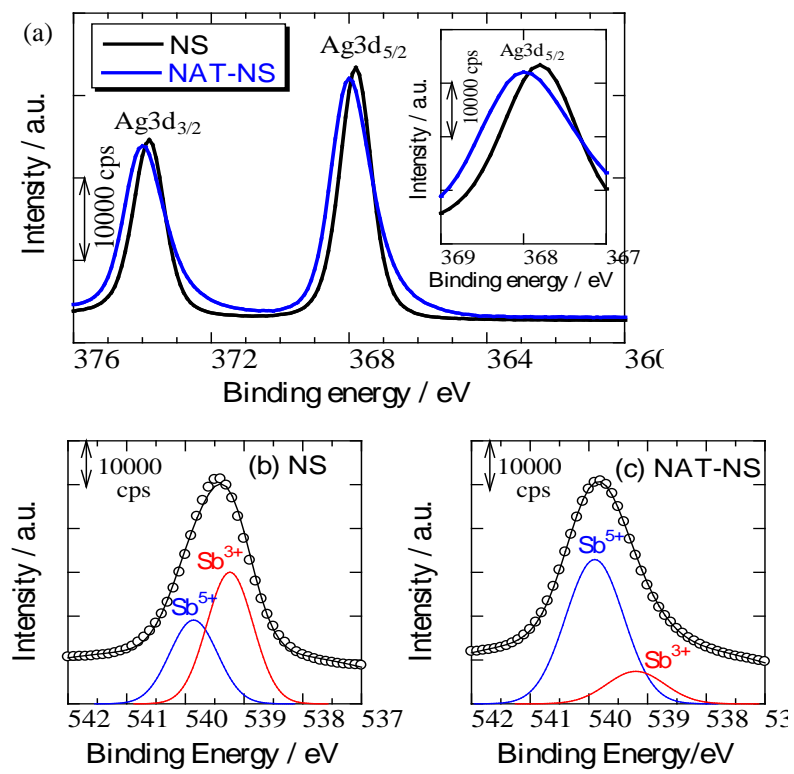

Figure 3. XPS spectra for (a) Ag $3 d$ ( $A g 3 d_{3 / 2}, A g 3 d_{5 / 2}$ ), Sb $3 d_{3 / 2}$ of (b) NS and (c) NAT-NS. The plotted experimental data and the fitted curves (black lines) closely coincided. The deconvolution curves of $\mathrm{Sb}^{3+}$ (red lines) and $\mathrm{Sb}^{5+}$ (blue lines) are included. The $\mathrm{Sb} 3 \mathrm{~d}$ peaks were calibrated using the $\mathrm{C} 1 \mathrm{~s}$ peak derived from the hydrocarbon surface contaminant with a binding energy of $284.6 \mathrm{eV}$. The $\mathrm{Sb}^{3+} / \mathrm{Sb}^{5+}$ ratios obtained from the peak deconvolution are described in Table 2.

in $\mathrm{AgSbO}_{3}$ including $\mathrm{Ag}^{0}$ and $\mathrm{Ag}^{2+}$ oxidation states. The $\mathrm{Sb}_{3} \mathrm{~d}_{3 / 2}$ XPS spectra of NS and NAT-NS are shown in Figure 3(b) and Figure 3(c), respectively. As for the oxidation states of $\mathrm{Sb}$, the peaks at 540.7 and $539.7 \mathrm{eV}$ are assigned to the $3 \mathrm{~d}_{3 / 2}$ orbital of $\mathrm{Sb}^{5+}$ and $\mathrm{Sb}^{3+}$, respectively [13]. To quantitatively evaluate the $\mathrm{Sb}^{3+} / \mathrm{Sb}^{5+}$ atomic ratios, peak deconvolution was performed using a Gaussian lineshape. The component $\mathrm{Sb}^{3+}$ and $\mathrm{Sb}^{5+}$ spectra determined by deconvolution and fitted peaks are also shown in Figure 3(b) and Figure 3(c). The atomic ra-

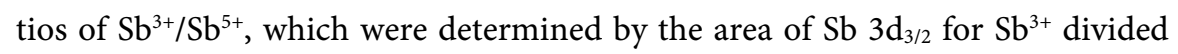
by thatfor $\mathrm{Sb}^{5+}$, are also shown in Table 2 . When we compare the $\mathrm{Sb}^{3+} / \mathrm{Sb}^{5+}$ ratio between NS and NAT-NS, the amount of $\mathrm{Sb}^{5+}$ increased after $\mathrm{HNO}_{3}$ treatment. This is also reasonable because $\mathrm{HNO}_{3}$ acts as an oxidant. So, it could be considered that $\mathrm{Ag}^{2+}$ also increased, and then $\mathrm{Ag}^{0}$ in $\mathrm{AgSbO}_{3}$ might increase to maintain charge neutrality (Figure $2 \&$ Figure 3 ). 


\subsection{Thermoelectric Properties}

Figure 4(a) shows the temperature dependence of $\sigma$ for NS and NAT-NS. The $\sigma$ values of both samples increased with increasing temperature, which is typical semiconductor behavior. In Figure 4(a), NS containing larger amount of $\mathrm{Sb}^{3+}$ with the similar amount of the separated Ag, compared to NAT-NS, indicated the higher $\sigma$. So, producing $\mathrm{Sb}^{3+}$ is the key to increase $\sigma$. This could be plausible because generating $\mathrm{Sb}^{3+}$ caused the generation of more oxygen vacancies in the material, and thus the corresponding donor levels are created in the band gap. This can provide more conduction electrons than in the case of no donor levels in the gaps. It should be noted that the $\mathrm{Sb}^{3+} / \mathrm{Sb}^{5+}$ ratio did affect $\sigma$ more greatly than the $\mathrm{Ag} / \mathrm{Sb}$ ratio. As mentioned above, NAT-NS containing Ag-defect $\left(\mathrm{Ag}_{0.98} \mathrm{Sb}_{1.00} \mathrm{O}_{y}\right)$ has more amounts $\mathrm{Ag}^{0}\left(4 \mathrm{~d}^{10} 5 \mathrm{~s}^{1}\right)$ and $\mathrm{Ag}^{2+}\left(4 \mathrm{~d}^{9}\right)$, meaning the in-

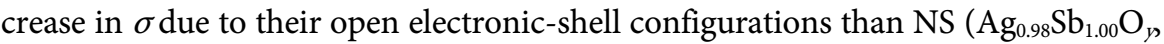
$\mathrm{Ag}^{+}\left(4 \mathrm{~d}^{10}\right)$, closed electronic-shell configuration). However, the opposite experimental results were observed.

Temperature dependence of $S$ for NS and NAT-NS is shown in Figure 4(b). $S$ values of both samples were negative, indicating the occurrence of n-type conduction. The trend of the absolute values of $S$ almost covers the Ioffe's theory, that is, the absolute value of $S$ decreases when $\sigma$ increases. So, NAT-NS had the higher absolute values of $S$. Figure 4(c) shows the temperature dependence of power factor $(P F)$, which was calculated from the formula $\sigma \times S^{2}$. The $P F$ observed in this study $\left(\sim 1 \times 10^{-5} \mathrm{~W} \cdot \mathrm{m}^{-1} \cdot \mathrm{K}^{-2}\right)$ was obtained for NS at $600^{\circ} \mathrm{C}$.

Finally, we examined the value of $Z T$ for the NS and NAT-NS samples at $600^{\circ} \mathrm{C}$ using the modified Harman method and then $\kappa$ was calculated. The $Z T$ values for NS and NAT-NS were 0.009 and 0.007 , respectively. The $\kappa$ value for NS was calculated to be $0.42 \mathrm{~W} \cdot \mathrm{m}^{-1} \cdot \mathrm{K}^{-1}$, which is reasonably consistent with the previously reported values of 1.1 and $0.8 \mathrm{~W} \cdot \mathrm{m}^{-1} \cdot \mathrm{K}^{-1}$ at room temperature and

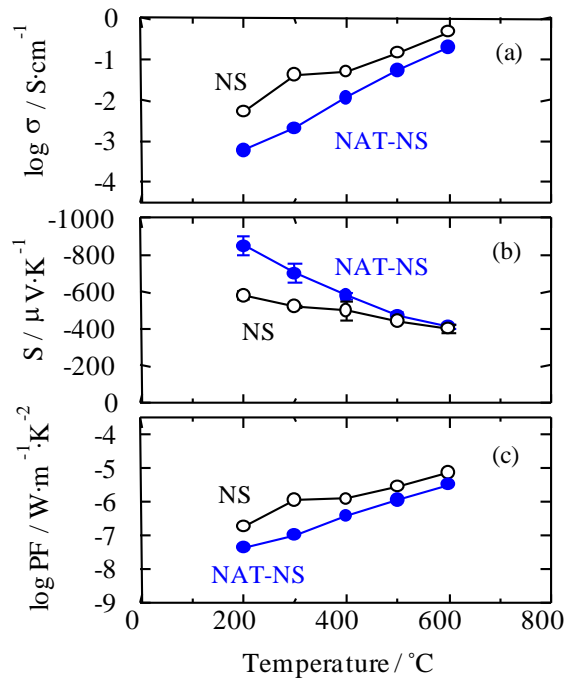

Figure 4. Temperature dependence of (a) electrical conductivity, $\sigma$ and (b) Seebeck coefficient, $S$, and (c) power factor $(P F)$ for NS and NAT-NS samples. 
$400^{\circ} \mathrm{C}$, respectively [8]. The $\kappa$ value for NAT-NS was $0.38 \mathrm{~W} \cdot \mathrm{m}^{-1} \cdot \mathrm{K}^{-1}$, which is slightly smaller than that for NS. This might be attributable to the presence of Ag defects, causing the phonon scattering by the defect site. Although these $Z T$ values need to be increased by around two orders of magnitude to be suitable for practical use, this study provides that the introduction of $\mathrm{Sb}^{3+}$ in $\mathrm{AgSbO}_{3}$ enhanced the thermoelectric property.

\section{Conclusion}

We prepared $\mathrm{AgSbO}_{3}$-based thermoelectric materials with and without the $\mathrm{HNO}_{3}$ treatment. Then the sample that had higher $\sigma$ indicated the higher $Z$, attributable to the generation of $\mathrm{Sb}^{3+}$, in spite of smaller $S$ and larger $\kappa$. The $Z T$ value for NS was observed to be $\sim 0.009$ at $600^{\circ} \mathrm{C}$, which is two orders of magnitude smaller than the minimum value considered being required for practical use, which is greater than 1 . To achieve a higher $Z T$ value, we are now trying to prepare dense $\mathrm{AgSbO}_{3}$ with and without $\mathrm{HNO}_{3}$ treatment using the SPS method because it is well-known that the SPS method allows the production of dense samples, resulting in the enhanced TE properties.

\section{Acknowledgements}

The experiments at SPring-8 were performed with the approval of the Japan Synchrotron Radiation Research Institute (JASRI) (Proposal No. 2014A1008).

\section{References}

[1] Terasaki, I., Sasago, Y. and Uchinokura, K. (1997) Large Thermoelectric Power in $\mathrm{NaCo}_{2} \mathrm{O}_{4}$ Single Crystals. Physical Review B, 56, R12685-R12687. https://doi.org/10.1103/PhysRevB.56.R12685

[2] Ohtaki, M., Tsubota, T., Eguchi, K. and Arai, H. (1996) High-Temperature Thermoelectric Properties of $\left(\mathrm{Zn}_{1-\mathrm{x}} \mathrm{Al}_{\mathrm{x}}\right) \mathrm{O}$. Journal of Applied Physics, 79, 1816.

[3] Li, S., Funahashi, R., Matsubara, I., Ueno, K., Sodeoka, S. and Yamada, H. (2000) Synthesis and Thermoelectric Properties of the New Oxide Materials $\mathrm{Ca}_{3-x} \mathrm{Bi}_{x} \mathrm{Co}_{4} \mathrm{O}_{9+\delta}(0.0<x<0.75)$. Chemistry of Materials, $12,2424-2427$. https://doi.org/10.1021/cm000132r

[4] Ohta, H., Kim, S., Mune, Y., Mizoguchi, T., Nomura, K., Ohta, S., Nomura, T., Nakanishi, Y., Ikuhara, Y., Hirano, M., Hosono, H. and Koumoto, K. (2007) Giant Thermoelectric Seebeck Coefficient of a Two-Dimensional Electron Gas in $\mathrm{SrTiO}_{3}$. Nature Materials, 6, 129-134. https://doi.org/10.1038/nmat1821

[5] Nishiyama, S., Ichikawa, A. and Hattori, T. (2004) Theromoelectric Properties of $\mathrm{CuO}$-Added $\mathrm{AgSbO}_{3}$ Ceramics. Journal of the Ceramic Society of Japan, 112, 298300. https://doi.org/10.2109/jcersj.112.298

[6] Allen, J.P., Nilsson, M.K., Scanlon, D.O. and Watson, G.W. (2011) Comparison of the Defective Pyrochlore and Ilmenite Polymorphs of $\mathrm{AgSbO}_{3}$ Using GGA and Hybrid DFT. Physical Review B, 83, Article ID: 035207.

https://doi.org/10.1103/PhysRevB.83.035207

[7] Sang, H.-Y. and Li, J.-F. (2010) Thermoelectric Properties of $\mathrm{AgSbO}_{3}$ with Defect Pyrochlore Structure. Journal of Alloys and Compounds, 493, 678-682.

https://doi.org/10.1016/j.jallcom.2009.12.190 
[8] Li, F. and Li, J.-F. (2011) Microstructure and Thermoelectric Properties of $\mathrm{AgSbO}_{3}$ Ceramics Prepared by Ion-Exchange Powder Synthesis and Normal Sintering. Journal of Electronic Materials, 40, 1035-1041. https://doi.org/10.1007/s11664-011-1525-0

[9] Wiggers, H., Simon, U. and Schon, G. (1998) Conductivity Studies on $\mathrm{AgSbO}_{3}$ Channel Structure by Impedance Spectroscopy. Solid State Ionics, 107, 111-116. https://doi.org/10.1016/S0167-2738(97)00518-3

[10] Kobayashi, R., Tanigawa, S., Takashima, T., Ohtani, B. and Irie, H. (2014) SilverInserted Heterojunction Photocatalysts for Z-Scheme Overall Pure-Water Splitting under Visible-Light Irradiation. The Journal of Physical Chemistry C, 118, 2245022456. https://doi.org/10.1021/jp5069973

[11] Kawano, T., Kakemoto, H. and Irie, H. (2015) Niobium(V) Oxide with Added Silver as a Thermoelectric Material Prepared by Spark Plasma Sintering. Materials Letters, 156, 94-97. https://doi.org/10.1016/j.matlet.2015.04.148

[12] Melin, E.P., Diaz, O.G., Rodrigues, J.M.D., Colon, G. and Navio, G.C. (2012) Effect of Deposition of Silver on Structural Characteristics and Photoactivity of $\mathrm{TiO}_{2}-$ Based Photocatalysts. Applied Catalysis B: Environmental,127, 112-120. https://doi.org/10.1016/j.apcatb.2012.08.007

[13] Zhang, H., Sun, K., Feng, Z., Ying, P. and Li, C. (2006) Studies on the $\mathrm{SbO}_{\mathrm{x}}$ Species of $\mathrm{SbO}_{\mathrm{x}} / \mathrm{SiO}_{2}$ Catalysts for Methane-Selective Oxidation to Formaldehyde. Applied Catalysis A: General, 305, 110-119. https://doi.org/10.1016/j.apcata.2006.02.038

Submit or recommend next manuscript to SCIRP and we will provide best service for you:

Accepting pre-submission inquiries through Email, Facebook, LinkedIn, Twitter, etc. A wide selection of journals (inclusive of 9 subjects, more than 200 journals) Providing 24-hour high-quality service User-friendly online submission system Fair and swift peer-review system Efficient typesetting and proofreading procedure Display of the result of downloads and visits, as well as the number of cited articles Maximum dissemination of your research work

Submit your manuscript at: http://papersubmission.scirp.org/ Or contact msce@scirp.org 